\title{
Kinder- und jugendpsychiatrische Versorgung 2019 in Österreich - Stufen der Versorgung, Ist-Stand und Ausblick
}

\author{
Rainer FliedI · Berenike Ecker · A. Karwautz (D)
}

Eingegangen: 8. Oktober 2020 / Angenommen: 27. Oktober 2020 / Online publiziert: 30. November 2020

(c) Der/die Autor(en) 2020

\section{Zusammenfassung}

Hintergrund Kinder- und jugendpsychiatrische Störungen sind häufig, die Versorgung in Österreich ist erst im Aufbau.

Methoden Wir untersuchten, ob sich die fachärztliche Versorgungslage seit Etablierung des Sonderfaches verbessert hat.

Ergebnisse Es besteht weiter eine große Heterogenität der Versorgungslage zwischen den Bundesländern, Die Mangelfachregelung in der derzeitigen Form ist nicht ausreichend, um die Ausbildung im Sonderfach zu konsolidieren und eine Vollversorgung in absehbarer Zeit zu erreichen.

Conclusion Es werden Anregungen erarbeitet, wie die Versorgungssituation in der Zukunft verbessert werden könnte.

Schlüsselwörter Versorgung · Österreich · Kinderund Jugendpsychiatrie

\footnotetext{
Dr. R. Fliedl

Lindauergasse 27/2, 1160 Wien, Österreich

rainer.fliedl@hotmail.com

Mag. B. Ecker, M.E.S., MSc

ÖGKJP, Wien, Österreich

sekretariat@oegkjp.at

Univ.-Prof. Dr. A. Karwautz, FAED ( $₫)$

Universitätsklinik für Kinder- und Jugendpsychiatrie,

General Hospital of Vienna, Medizinische Universität Wien,

Währinger Gürtel 18-20, 1090 Wien, Österreich

andreas.karwautz@meduniwien.ac.at
}

Child and adolescent psychiatric care 2019 in Austria-steps of care, current state and lookout

\section{Summary}

Background Child and adolescent psychiatric disorders are prevalent, their care in Austria is still in progress.

Methods We investigated whether the child and adolescent psychiatric care made progress dsince the establishment of the medical special discipline.

Results There remains massive heterogeneity between the Austrian provinces. The "Mangelfachregelung" in ist current version is insufficient in order to consolidate our medical special discipline and to guarantee full carewithin the next years.

Conclusion Hints are given how the situation of care in our discipline can be strengthened.

Keywords Care - Austria - Child and Adolescent Psychiatry

\section{Einleitung}

Durch die Österreichische Gesellschaft für Kinderund Jungendpsychiatrie, Psychosomatik und Psychotherapie (ÖGKJP) werden seit vielen Jahren die landesweiten Versorgungsdaten erhoben und ausgewertet [1-3]. Auf Basis der gewonnenen Ergebnisse werden Empfehlungen für die Weiterentwicklung der kinder- und jugendpsychiatrischen Versorgung (KJPVersorgung) in Österreich abgeleitet. Die vorliegende Arbeit beinhaltet Daten aus den Jahren 2012 bis 2019. Die Daten für 2019 (mit Stichtag 31.12.2019) wurden im Frühjahr 2020 bei den Vorständen der jeweiligen Abteilungen erhoben.

In der vorliegenden Arbeit folgt zuerst ein Überblick zum Versorgungskonzept und den Stufen der Versorgung. In weiterer Folge werden die Bereiche sta- 
tionäre Versorgung, ambulante Versorgung und Versorgung durch den niedergelassenen Bereich entlang der erhaltenen Daten beschrieben, die Ausbildungssituation diskutiert und auf innovative und kooperative Aspekte im Versorgungskontext hingewiesen.

\section{Epidemiologie psychischer Störungen in Österreich und quantitative Annäherung an die notwendigen Behandlungsplätze}

In der für Österreich repräsentativen Erhebung zu psychischen Störungen gemäß DSM-5 bei 10-18jährigen Jugendlichen, der MHAT-Studie („Mental Health in Austrian Teenagers") fanden wir [4] eine Halbjahresprävalenz von 23,9\% psychischer Störungen sowie eine Lebenszeitprävalenz von $35,8 \%$. Fast jede(r) vierte Jugendliche leidet aktuell unter einer psychischen Störung. Per definitionem bedarf jede psychische Krankheit einer Behandlung, allerdings nicht jede einer akuten und unmittelbaren Intervention. Angelehnt an die Kriterien von Packard [5] (Guidelines to use of axis V) besteht bei einem GAF („Global Assessment of Functioning“) von größer/ gleich 70 Behandlungsbedürftigkeit, bei GAF Werten unter 70 akute Behandlungsbedürftigkeit. Wir konnten für die Gesamtstichprobe für $8 \%$ der österreichischen jugendlichen Population ( $n=780.185$; 10-18 Jahre; Statistik Austria 2014) eine DSM-5 Diagnose und einen GAF von über 70 feststellen, für $14 \%$ bei Vorliegen einer Diagnose einen GAF von kleiner als $70.78 \%$ der Jugendlichen waren seelisch gesund.

Das bedeutet in Zahlen: 170.800 Jugendliche in Österreich leiden 2014 an einer psychischen Störung, davon sind 106.800 akut behandlungsbedürftig. Pro 1000 Jugendliche sind das 171 Menschen (20 pro 1000 Einwohner*innen Österreich) mit einer psychischen Krankheit, 107 davon (12,5 von 1000 Einwohner*innen in Österreich) wären akut behandlungsbedürftig.

Da durchschnittlich über alle Diagnosen knapp die Hälfte der in der MHAT-Studie identifizierten Jugendlichen mit einer Erkrankung bisher nirgends wegen dieser vorstellig wurden und keine adäquate Behandlung aufgesucht haben besteht hier eine hohe Dunkelziffer zu behandelnder Betroffener.

\section{Versorgungsprinzipien, Versorgungskonzept und Stufen der Versorgung}

Kinder- und jugendpsychiatrische und -psychotherapeutische Versorgung (KJPP-Versorgung) folgt dem Prinzip der Gemeindenähe und der Grundregel ambulant vor stationär [6]. Um dieses gestufte Versorgungsmodell wirksam aufzubauen, bedarf es einer definierten und differenzierten Beschreibung der einzelnen Bausteine der Versorgung und regional wirksamer Modelle der Kooperation. Um diese Kooperation $\mathrm{zu}$ ermöglichen, ist es notwendig, die einzelnen Bausteine quantitativ und qualitativ mit den dafür notwendigen Ressourcen auszustatten.
Eine wirksame und effiziente Gestaltung dieses gestuften Versorgungsmodells samt Berechnungsschlüssel [6] findet sich in Tab. 1. Es bedarf zudem regional wirksamer Modelle der Kooperation der mit der Behandlung und Betreuung von Kindern und Jugendlichen verantworteten Institutionen.

Im Kernbereich der KJPP-Versorgung sind im stationären Bereich psychosomatische und sozialpädiatrische Plätze und im ambulanten Bereich neuropädiatrische und psychotherapeutische Ambulanzen abzugrenzen und kooperativ weiter zu entwickeln. Auch die anderen Einrichtungen im Komplementärbereich im Rahmen der Jugendhilfe, der Schule und anderer Institutionen, die Kindern und Jugendlichen zur Verfügung gestellt werden, werden nicht im Detail beschrieben. Es bedarf aber einer gemeinsamen Planung und Weiterentwicklung um optimale Synergien in den überlappenden Aufgabenbereichen zu erzielen.

Der Berechnungsschlüssel der Bausteine bezieht sich auf den Bevölkerungsstand des Jahres 2019, dieser verändert sich kontinuierlich. 2019 hatte Österreich 8.901.064 (Statistik Austria 2020) [7] Einwohner*innen (Ew.) mit einem Anteil von 19,3\% Minderjährigen, das entspricht einem Bevölkerungswachstum von 0,5\% im Jahr 2019 [12]. Für das Jahr 2030 ist die Bevölkerungsprognose bei 9.210.146 Ew. (Statistik Austria 2020 [13]), der Anteil an Minderjäh-

Tab. 1 Bausteine der KJPP-Versorgung in Relation zu Einwohner*innen

\begin{tabular}{|c|c|c|}
\hline & Bausteine der Versorgung & $\begin{array}{l}\text { Berechnungs- } \\
\text { schlüssel }\end{array}$ \\
\hline \multirow[t]{5}{*}{ Stationär } & $\begin{array}{l}\text { Intensivbehandlung } \\
\text { Behandlungsform „I“ im LKF-System }\end{array}$ & \multirow{5}{*}{$\begin{array}{l}0,1 \text { pro } 1000 \\
\text { Einwoh- } \\
\text { ner*innen }\end{array}$} \\
\hline & $\begin{array}{l}\text { Behandlungsplätze } \\
\text { Behandlungsform „A“ im LKF-System }\end{array}$ & \\
\hline & $\begin{array}{l}\text { Eltern-Kind-Plätze } \\
\text { Behandlungsform „E“ im LKF System }\end{array}$ & \\
\hline & $\begin{array}{l}\text { Tagesklinische Behandlung } \\
\text { Behandlungsform „T“ im LKF-System }\end{array}$ & \\
\hline & $\begin{array}{l}\text { Mobile Plätze } \\
\text { Home-Treatment } \\
\text { integrative Versorgung } \\
\text { Fehlen im LKF-System }\end{array}$ & \\
\hline \multirow[t]{2}{*}{ Ambulant } & Ambulanzen oder Ambulatorien & $\begin{array}{l}\text { Pro } 250.000 \\
\text { Einwoh- } \\
\text { ner*innen }\end{array}$ \\
\hline & Niedergelassene Fachärztinnen & $\begin{array}{l}\text { Pro } 80.000 \\
\text { auf Einwoh- } \\
\text { ner*innen }\end{array}$ \\
\hline \multirow[t]{3}{*}{$\begin{array}{l}\text { Komplementär- } \\
\text { bereich }\end{array}$} & $\begin{array}{l}\text { Beratungsstelle mit KJPP-Kompetenz } \\
\text { für Kinder und Jugendliche und ihre } \\
\text { Familien }\end{array}$ & $\begin{array}{l}\text { Pro } 80.000 \text { Ein- } \\
\text { wohner*innen }\end{array}$ \\
\hline & $\begin{array}{l}\text { Ambulante und stationäre Einrichtun- } \\
\text { gen der Jugendhilfe }\end{array}$ & - \\
\hline & $\begin{array}{l}\text { Einrichtungen im Bildungssystem und } \\
\text { des Arbeitsmarktservices (AMS) wie } \\
\text { Beratungslehrer*innen, Schulsozialar- } \\
\text { beit, Sonderkindergärtner*innen }\end{array}$ & - \\
\hline Partizipation & Betroffenen- und Elternvertretungen & - \\
\hline
\end{tabular}


Tab. 2 Bedarf bezogen auf die österreichische Bevölkerung

\begin{tabular}{|c|c|c|}
\hline Einrichtungen & Berechnungsschlüssel & Bedarf \\
\hline $\begin{array}{l}\text { Stationäre und tagesklinische Plätze } \\
\text { (BMZ) }\end{array}$ & 0,1 pro $1000 \mathrm{Ew}$ & 890 \\
\hline Ambulanzen oder Ambulatorien & 1 pro $250.000 \mathrm{Ew}$ & 36 \\
\hline Niedergelassene Fachärzt*innen & 1 pro 80.000 auf Ew & 111 \\
\hline
\end{tabular}

rigen von 19,3\% wird gleichbleiben. Dies entspricht einem Wachstum von 5\%. In dieser Arbeit wird die IST-SOLL Divergenz von 2019 angesprochen, sinnvolle Planungszahlen liegen in einzelnen Regionen bis zu $10 \%$ über diesen Werten.

Diese spezifischen Versorgungsangebote und -einrichtungen sind andernorts [8-10] bereits ausführlich beschrieben und über Qualitätsstandards definiert.

Aus den Daten der MHAT-Studie ist ersichtlich, dass in einer Standardversorgungsregion von 500.000 Einwohner*innen (Ew.) 6250 Kinder und Jugendliche akut behandlungsbedürftig (GAF unter 70) sind.

Quantitativ lässt sich der Bedarf in einer Standardversorgungsregion wie folgt zusammenfassen:

- Eine stationäre Einrichtung für KJPP mit 50 Behandlungsplätzen $(0,1 \% 0)$,

- Zwei kinder- und jugendpsychiatrische Ambulanzen bzw. Ambulatorien,

- Sechs bis sieben niedergelassene Fachärzt*innen für Kinder- und Jugendpsychiatrie,

- Sechs bis sieben Miniambulatorien/Beratungsstellen mit kinder- und jugendpsychiatrischer Kernkompetenz [11].

Bezogen auf das Bundesgebiet Ö ergibt sich der in Tab. 2 dargelegte Bedarf.

\section{Versorgungslage in Österreich}

\section{Stationärer Bereich}

2019 hatte Österreich 8.901.064 Einwohner*innen [14]. Die Mitversorgung von bayerischen Patient*innen durch Salzburg und von Patient*innen aus Liechtenstein durch Vorarlberg wird nicht eingerechnet.

Zur stationären kinder- und jugendpsychiatrischen Versorgung stehen zur Zeit 12 Krankenhausabteilungen zur Verfügung. Von diesen Abteilungen haben sechs dislozierte Außenstellen mit einer Ambulanz und fünf dislozierte Außenstellen mit tagesklinischen Plätzen. Weiters gibt es die Ambulanz und Tagesklinik des Psychosozialen Dienstes (PSD) Wien.

Die Klinik Floridsdorf wird, da sie im Aufbau begriffen ist und mit 31.12.2019 nicht eigenständig versorgungswirksam ist, im Verbund mit dem der Abteilung für Kinder- und Jugendpsychiatrie und Psychotherapie (KJPP) der Klinik Hietzing beschrieben.

Die Versorgungsregionen haben eine Größe zwischen 397.139 Einwohner*innen (Vorarlberg) und
1.490.279 Einwohner*innen (Oberösterreich) (siehe auch Karte 1). Eine Standardversorgungsregion wird mit 500.000 Einwohner*innen gerechnet [11].

Besonders in flächenmäßig großen Versorgungsregionen wie beispielsweise Oberösterreich und Steiermark wären weitere Standorte notwendig, um eine Regionalisierung der Versorgung zu erzielen.

Seit 2016 kam es österreichweit zu einem Zuwachs von 119 Behandlungsplätzen. Davon waren 38 Plätze Stationär und 81 Tagesklinisch. Siehe auch Abb. 1. Dieser Zuwachs wurde durch folgende Erweiterungen erzielt.

- Niederösterreich: Die Eröffnung der Außenstellen der Krankenhausabteilungen Mistelbach, Wiener Neustadt und Waidhofen an der Thaya und den Neubau der KJPP im Landeskrankenhaus Mauer.

- Salzburg: Der Neubau der Uniklinik, die Eröffnung der KJPP im Krankenhaus Schwarzach

- Steiermark: Die tagesklinischen Plätze im Krankenhaus Leoben,

- Tirol: Der Neubau der KJPP im Landeskrankenhaus Hall und die Tagesklinik in Innsbruck.

- Wien: Die Plätze für die tagesklinische Behandlung an der Klinik Floridsdorf und eine Erhöhung der Plätze an der Klinik Hietzing.

Mit dem Zuwachs der Plätze hat sich die Bettenmessziffer (BMZ) in den letzten drei Jahren von 0,05auf 0,06 erhöht. Unter der BMZ versteht man die Zahl der Behandlungsplätze, die pro 1000 Einwohner benötigt wird, um eine ausreichende Versorgung zu gewährleisten. Der Wert von 0,1 wurde in Salzburg und Vorarlberg nahezu erreicht. Die niedrigsten Bettenmessziffern finden sich in der Steiermark und Wien, im Burgenland besteht keine eigene stationäre Versorgung, es besteht aber eine Mitversorgung durch Niederösterreich und die Steiermark und wird dort mitberechnet (Abb. 2).

\section{Tageskliniken}

Das Verhältnis von tagesklinischen zu vollstationären Plätzen fällt im Jahr 2019 österreichweit mit einem Verhältnis von $30 \%$ zu $70 \%$ relativ homogen aus. Im Vergleich zu 2018, wo das Verhältnis bei $27 \%$ zu $73 \%$ lag, hat der Anteil an tagesklinischen Betreuungsmöglichkeiten leicht zugenommen. Tirol stellt eine Ausnahme dar, da es dort bis auf die Ambulanz in Innsbruck noch keine dislozierten Einrichtungen gibt. Siehe auch Abb. 3.

\section{Home-Treatment und Eltern-Kind-Plätze}

Eltern-Kind-Aufnahmen sind in einigen Abteilungen (z. B. LKH Hall, LKH Mauer) möglich, in der Gesamtheit der Versorgung aber noch keine verlässliche Säule.

Für den Bereich des Home-Treatments wurden Konzepte erstellt. Vorbilder dafür gibt es z.B. in der 
Anzahl Plätze

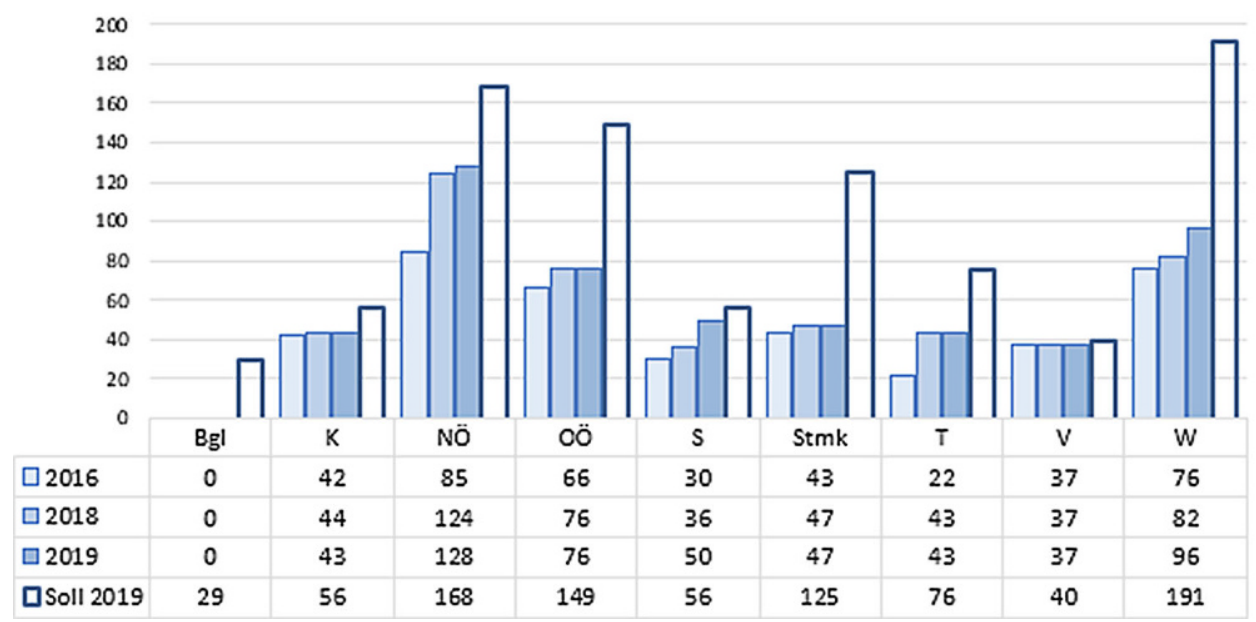

Anzahl Plätze

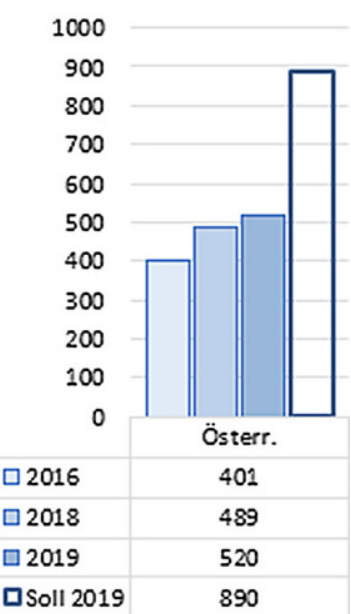

Abb. 1 Entwicklung der stationären und tagesklinischen Plätze 2016-2019 auf Bundeslandebene und für Gesamtösterreich

Abb. 2 Bettenmessziffer im Vergleich (2016, 2018 und 2019)
Bettenmessziffer

$\square 2016 \square 2018 \square 2019$

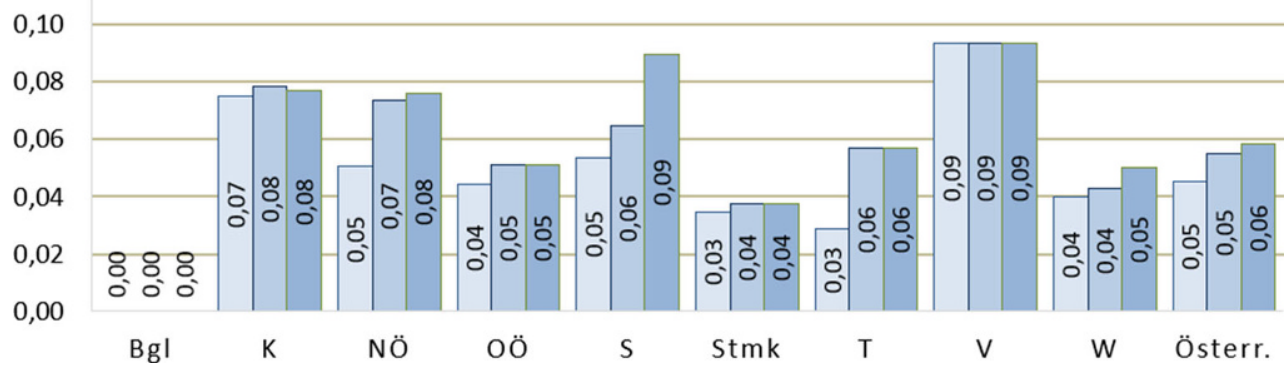

Verhältnis Stationär/TKL 2019

口TKL घstationär

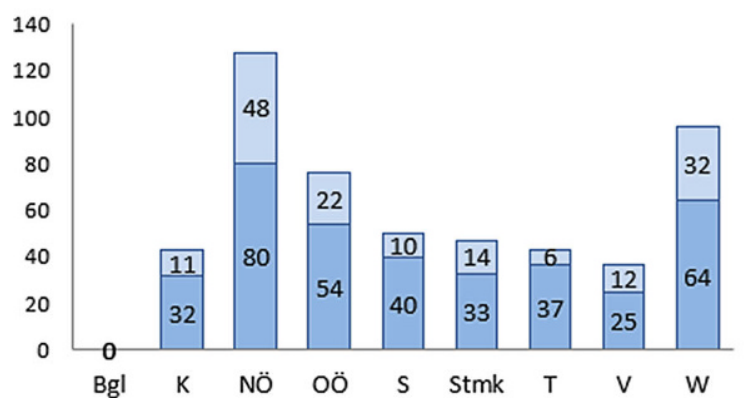

Verhältnis Stationär/TKL 2019 in \%

口stationär $\square \mathrm{TKL}$

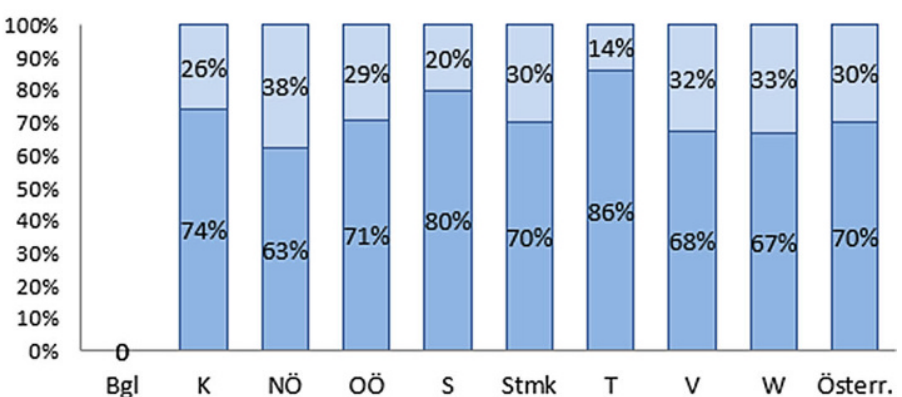

Abb. 3 Verhältnis tagesklinische zu vollstationären Plätzen im Jahr 2019 - Absolutzahlen sowie in \%

Kinder- und Jugendpsychiatrie Hamburg für Psychosen und in Aachen für Essstörungen. Es bedarf aber sowohl einer Verankerung im LKF-System als auch einer praktischen Umsetzung in den Einrichtungen.
Stationäre kinder- und jugendpsychiatrische Versorgungsregionen

Da die Versorgungsregionen nicht an die Bundesländergrenzen orientiert sind, ist eine Darstellung (siehe Abb. 4) der KJPP-Versorgungsregionen sinnvoll. Einige Bundesländer haben sinnvollerweise mehrere Versorgungsregionen. Patient*innen vom Nord- und Mittel- 


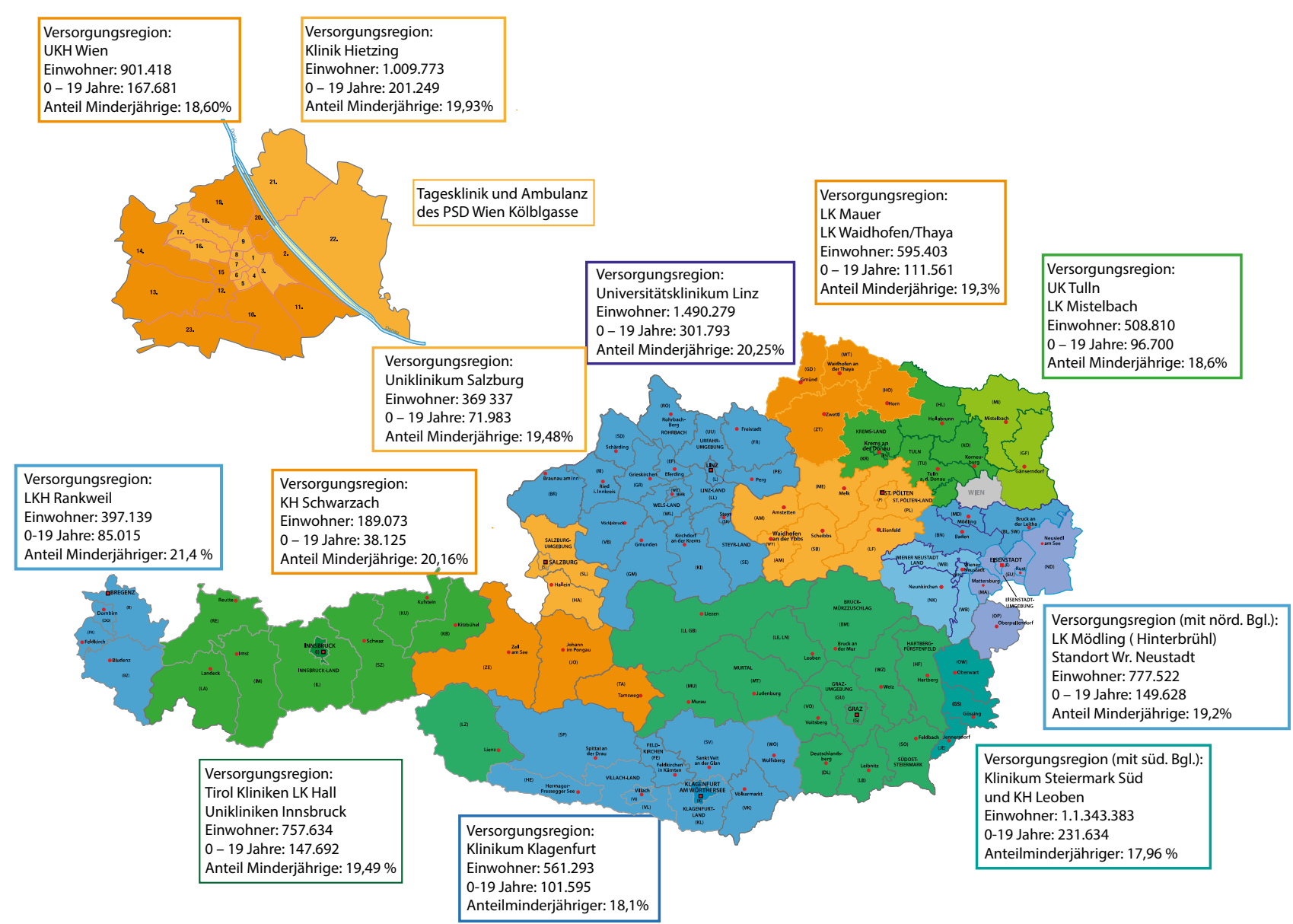

Abb. 4 Kinder- und jugendpsychiatrische Versorgungsregionen stationärer Bereich (Stand: 31.12.2019)

burgenland werden von der KJPP Hinterbrühl mitversorgt, jene vom südlichen Burgenland von der KJPP in Graz. Gewissermaßen vergleichbar stellt sich die Situation in Osttirol, das zum Teil von Kärnten mitversorgt wird. Zwischen den einzelnen Bundesländern ist daher zu klären, welche Landesregierung die Verantwortung für die Versorgung dieser Regionen übernimmt und vorantreibt.

Die stationäre und tagesklinische Versorgung auf der Ebene der Versorgungsregionen wird in Tab. 3 dargestellt. Neben der Einwohnerzahl und dem Anteil der Minderjährigen werden der Soll-stand mit dem Iststand verglichen und in der jeweils erreichten Bettenmessziffer als wesentlichster Bezugsgröße zusammengefasst.

\section{Ambulante Versorgung}

Wie in den Versorgungsprinzipien angesprochen, stellen sowohl Ambulanzen und Ambulatorien, als auch Niedergelassene wichtige Elemente der Versorgung dar, da über diese unterschiedlichen Angebote verschiedene Zugänge zur kinder- und jugendpsychiatrischen Versorgung geschaffen werden und damit eine höhere Versorgungsprävalenz erreicht werden kann.

\section{Ambulatorien und Ambulanzen}

Grundsätzlich ist eine ambulante Einrichtung für 250.000 Einwohner*innen notwendig. Dies entspricht für Gesamtösterreich 36 Ambulanzen oder Ambulatorien. Ein exakter Vergleich der Ambulanzen ist nicht möglich, da die zur Verfügung stehenden Personalressourcen der einzelnen Ambulanzen und damit ihre Versorgungskapazität unterschiedlich sind.

Zum Stichtag am 31.12.2019 gab es 22 ambulante Einrichtungen in Österreich. Hier werden die Boje und Extended Soulspace nicht eingerechnet, da sie spezifische Aufgaben und Aufträge haben. Diese Einrichtungen werden bei Spezialambulanzen beschrieben.

Wie aus Tab. 4 hervorgeht, besteht im Burgenland und in Salzburg eine gute Ausstattung mit Ambulanzen und/oder Ambulatorien. Der eklatanteste Mangel besteht in Wien, Oberösterreich und in der Steiermark.

\section{Spezialisierte Angebote an den Ambulanzen und Ambulatorien}

Trotz des erheblichen Mangels an der Zahl ambulanter Einrichtungen wurde eine spezifische Versor- 
Tab. 3 Versorgungsübersicht auf Ebene der Versorgungsregionen (Stand: 31.12.2019)

\begin{tabular}{|c|c|c|c|c|c|c|c|c|c|}
\hline Versorgungsregionen & Einwohner & $\begin{array}{l}\text { Anzahl } \\
\text { stationär }\end{array}$ & $\begin{array}{l}\text { Anzahl } \\
\text { TKL }\end{array}$ & $\begin{array}{l}\text { Summe } \\
\text { Stat. + TKL }\end{array}$ & $0-19$ & $\begin{array}{l}\text { \% Minder- } \\
\text { jährige }\end{array}$ & Soll Plätze & $\begin{array}{l}\text { Fehlende } \\
\text { Plätze }\end{array}$ & $\begin{array}{l}\text { Bettenmess- } \\
\text { ziffer }\end{array}$ \\
\hline Klinikum Klagenfurt/W & 561.293 & 32 & 11 & 43 & 101.595 & 18,10 & 56 & 13 & 0,077 \\
\hline LK Mauer & 595.403 & 30 & 16 & 46 & 118.014 & 19,82 & 60 & 14 & 0,077 \\
\hline LK Mödling (Hinterbrühl) & 777.522 & 30 & 16 & 46 & 149.628 & 19,24 & 78 & 32 & 0,059 \\
\hline UK Tulln & 508.810 & 20 & 16 & 36 & 96.700 & 19,01 & 51 & 15 & 0,071 \\
\hline UK Linz & 1.490 .279 & 54 & 22 & 76 & 301.793 & 20,25 & 149 & 73 & 0,051 \\
\hline Klinikum Salzburg & 369.337 & 30 & 10 & 40 & 71.983 & 19,49 & 37 & -3 & 0,108 \\
\hline Krankenhaus Schwarzach & 189.073 & 10 & 0 & 10 & 38.125 & 20,16 & 19 & 9 & 0,053 \\
\hline Stmk Standort Süd & 1.343 .383 & 33 & 14 & 47 & 241.440 & 17,97 & 134 & 87 & 0,035 \\
\hline LKH Hall & 757.634 & 37 & 6 & 43 & 147.692 & 19,49 & 76 & 33 & 0,057 \\
\hline LKH Rankweil & 397.139 & 25 & 12 & 37 & 167.681 & 21,41 & 40 & 3 & 0,093 \\
\hline UK Wien & 901.418 & 28 & 8 & 36 & 167.681 & 18,60 & 90 & 54 & 0,040 \\
\hline $\begin{array}{l}\text { Klinik Hietzing und Klinik Florids- } \\
\text { dorf und PSD Kölblgasse }\end{array}$ & 1.009 .773 & 36 & 24 & 60 & 201.249 & 19,93 & 101 & 65 & 0,059 \\
\hline
\end{tabular}

Tab. 4 Versorgung durch Ambulanzen und/oder Ambulatorien im Jahr 2019. Soll-Ist-Vergleich

\begin{tabular}{|l|l|l|}
\hline & Soll & Ist \\
\hline Burgenland & 1 & 2 \\
\hline Kärnten & 2 & 1 \\
\hline Niederösterreich & 7 & 5 \\
\hline Oberösterreich & 6 & 1 \\
\hline Salzburg & 2 & 3 \\
\hline Steiermark & 5 & 2 \\
\hline Tirol & 3 & 2 \\
\hline Vorarlberg & 2 & 2 \\
\hline Wien & 8 & 4 \\
\hline Österreich & 36 & 22 \\
\hline
\end{tabular}

gung für unterschiedliche Patient*innengruppen aufgebaut, in denen, den Ressourcen entsprechend, spezifische Behandlungsangebote erteilt werden können. Viele dieser Angebote sind an mehreren Abteilungen möglich, ein Schwerpunkt liegt an den Universitätskliniken (alphabetisch).

- ADHS

- Autismus

- Drogen

- EEG

- Entwicklung

- Essstörungen

- Forensik

- Genderinkongruenz

- Geschlechtsdysphorie

- Kinder psychiatrisch kranker Eltern

- Kinder suchtkranker Eltern

- Psychologische Diagnostik

- Psychose-Nachsorge Psychotherapie

- Psycho-Diabetologie

- Säugling/Kleinkind

- Schmerz
- Tourette Syndrom

- Transkulturelle Probleme

- Traumafolgestörungen

- UMF (unbegleitete minderjährige Flüchtlinge)

\section{„Spezialambulanzen“}

\section{- Ambulatorium die Boje}

Die Boje hat einen spezifischen Versorgungsauftrag in der interdisziplinären Versorgung akut traumatisierter Kinder und Jugendlicher. Die Versorgungsregion ist Wien, Niederösterreich und das Burgenland. Die Ausstattung der Einrichtung entspricht den Standards eines Ambulatoriums [10] Durch den niederschwelligen Zugang und der Möglichkeit zu Akutinterventionen können Kinder und Jugendliche in traumatisierenden Situationen in kurzer Zeit Hilfe bekommen.

- Kinder- und Jugendpsychiatrische Ambulatorium mit Tagesklinik-Extended Soulspace des PSD Wien Das Ambulatorium hat mit der Versorgung von Kindern und Jugendlichen im Rahmen von Maßnahmen der Jugendhilfe einen spezifischen Versorgungsauftrag. Neben der ambulanten Behandlung von Kindern und Jugendlichen werden auch Hausbesuche in Wohngemeinschaften gemacht, weiters stehen 16 Plätze für tagesklinische Behandlung zur Verfügung. Die Einrichtung ist ein wichtiges Behandlungsangebot für ein besonders gefährdetes Klientel.

\section{Nebentätigkeiten der Ambulanzen}

Neben der primären ambulanten Versorgungstätigkeit werden Konsiliardienste für andere Abteilungen (Erwachsenenpsychiatrie, Pädiatrie, Drogenstation, Kinderchirurgie, Jugendforensik, Onkologie, Transplant, Intensiv, Neonatologie), Konsiliar- und Liaisondienste 
für Krisenzentren, Wohngemeinschaften und Heime der Jugendhilfe, und KJPP-Versorgung der unbegleiteten minderjährigen Flüchtlinge angeboten. Für diese Leistungen stehen nur an wenigen Abteilungen gesonderte Personalressourcen zu Verfügung. Gleichzeitig sind diese Aufgaben von großer Relevanz, da dadurch die die psychiatrische Versorgung in diesen Bereichen verbessert werden kann.

\section{Niedergelassene Kassenärzt*innen}

Seit 2016 kam es österreichweit zu einem Zuwachs von 4,5 Kassenplätzen. Damit wurden $36 \%$ der für die Versorgung notwendigen Kassenplätze geschaffen. Besonders bedauerlich ist, dass es im Burgenland und in der Steiermark keine Kassenordinationen gibt und dies auch eine strukturelle Schlechterstellung der $\mathrm{Pa}$ tient*innen in diesen Bundesländern zur Folge hat. Siehe auch Abb. 5.

\section{Die Fachärzt*innenausbildung im Fach Kinder- und Jugendpsychiatrie und Psychotherapeutische Medizin}

Die problematische Situation des Mangels an Fachärzt*innen wurde von Hartl und Karwautz [2] differenziert beschrieben. In dieser Arbeit wird ausgehend von 2013 verdeutlicht, dass es unter günstigen Bedingungen mehr als 10 Jahre dauern wird, um die erforderliche Anzahl an Fachärzt*innen auszubilden (siehe auch Abb. 6).

Es absolvierten in den Jahren 2015 bis 2019 zwischen neun und fünfzehn Ärzt*innen die Facharztprüfung, damit konnte bei einem Durchschnitt von 12 die Untergrenze der Prognose nicht erreicht werden. Die Entwicklung der letzten Jahre lässt eine günstige Prognose (20 oder 15 Anerkennungen pro Jahr) bis jetzt daher nicht erwarten, das Fach stagniert also zahlenmäßig und das Erreichen der Vollversorgung ist jetzt absehbar (Stand 2019/20) weiter nicht in Aussicht.
Es bleibt daher wichtig, eine größere Anzahl an Assistenzärzt*innen auszubilden, um dem Bedarf an kinder- und jugendpsychiatrischer Versorgung quantitativ Rechnung zu tragen und auch die damit in Verbindung stehende qualitative Weiterentwicklung des Faches voranbringen zu können.

Mit dem Stichtag 31.12.2019 gab es an den Befragten Einrichtungen 96 genehmigte Ausbildungsstellen. Diese geringe Anzahl kann auch durch die Mangelfachregelung in der ÄAÖ $2015 \$ 37$ (1) die bis 31.05.2021 gültig ist, nicht deutlich verbessert werden. Eine Verbesserung wäre mit der Regelung „Eine Fachärzt*in kann zwei Assistent*innen ausbilden“ möglich.

Wegen des Mangels an Fachärzt*innen konnten österreichweit von den 125 Dienstposten für Fachärzt*innen 25 Dienstposten nicht besetzt werden. Da diese Facharztstellen auch in den nächsten Jahren nur sehr langsam besetzbar sind, wird es zu keiner Vermehrung der Ausbildungsplätze kommen.

Wir können daher mit etwa 19 neuen Fachärzt*innen pro Jahr rechnen (entsprechend der Anzahl der Absolventen der Fachärzt*innenprüfung). Da circa 1/3 der neuen Fachärzt*innen nicht im öffentlichen $\mathrm{Ge}$ sundheitsbereich arbeiten wird, sondern in privaten Ordinationen (welche üblicherweise nicht voll versorgungsrelevant sind), können wir zur Zeit nur mit einem Zuwachs von 12-13 Fachärzt*innen rechnen, die in komplett versorgungsrelevanten Strukturen tätig werden - diese Anzahl wird in den nächsten Jahren gerade die Anzahl an Fachärzt*innen, die in Pension gehen, decken.

Zum Stichtag waren 4 Ausbildungsstellen nicht besetzt. Dies ist nachvollziehbar, da zu diesem Zeitpunkt an zwei Abteilungen die Ausbildungsstellen nicht durch Fachärzt*innen abgedeckt wurden und daher nicht besetzbar waren und an einer Abteilung keine Dienstposten zur Verfügung standen um Assistenzärzt*innen anzustellen.

Eine Vermehrung der Ausbildungsstellen ist daher primär nur durch eine Veränderung der Mangelfach-
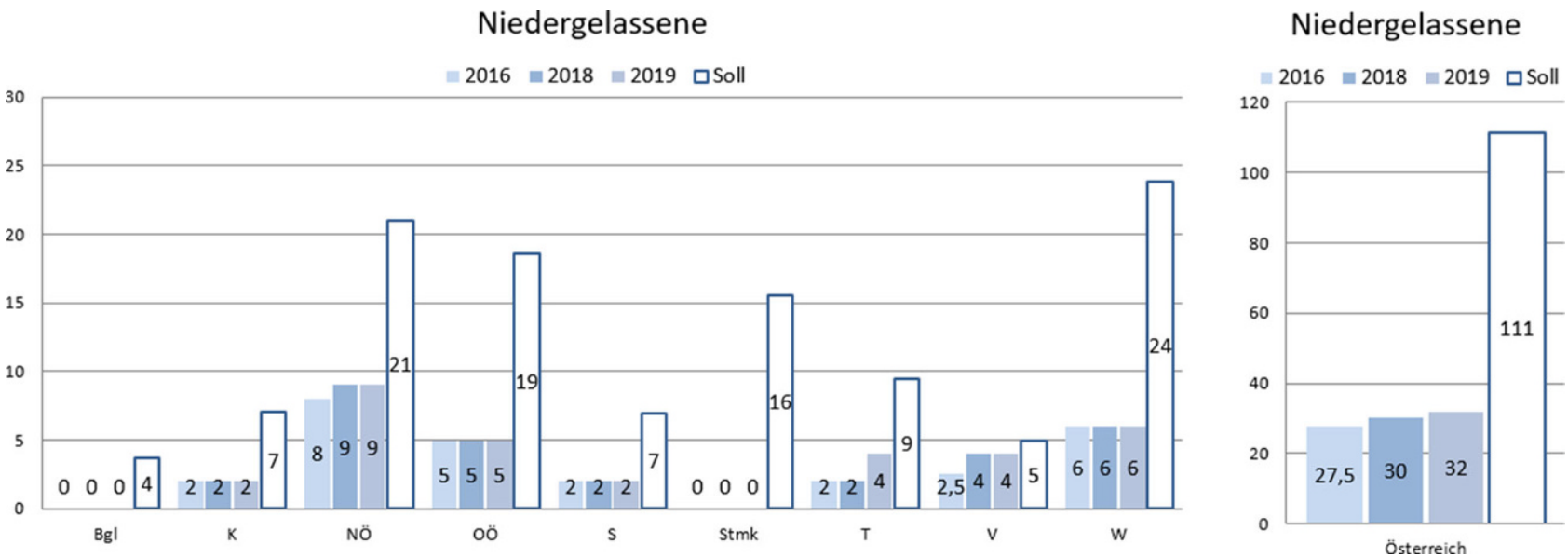

Abb. 5 Niedergelassene im Vergleich (2016, 2018 und 2019 bzw. Sollzahlen). Quelle: [10] 


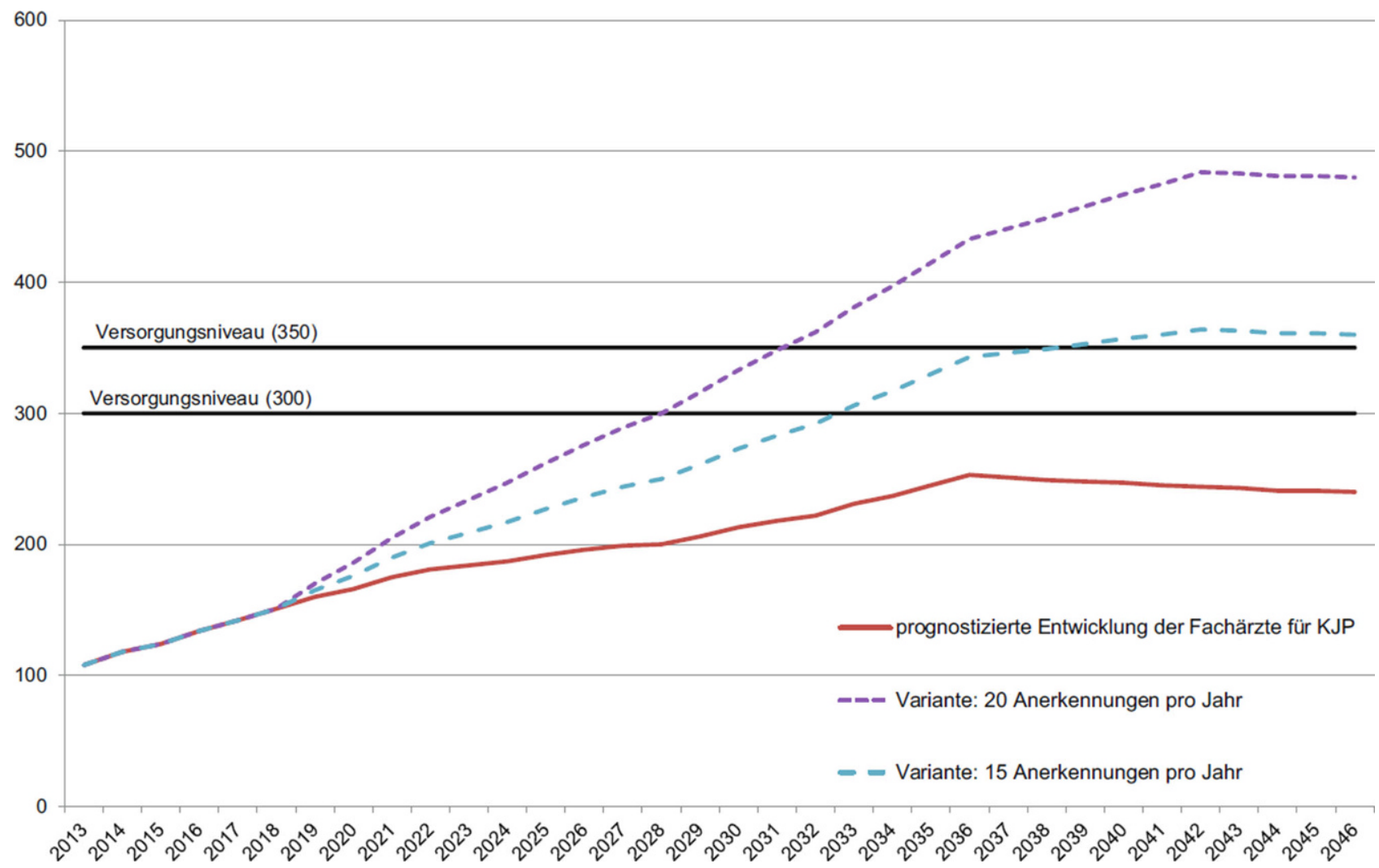

Abb. 6 Prognose der Facharztentwicklung mit 2013 (alte ÄAO). Quelle: Hartl und Karwautz [2]

Abb. 7 Verteilung Ärzt*innen und Psycholog*innen in \% im Jahr 2019
Verteilung Ärzt*innen und Psycholg*innen in \%
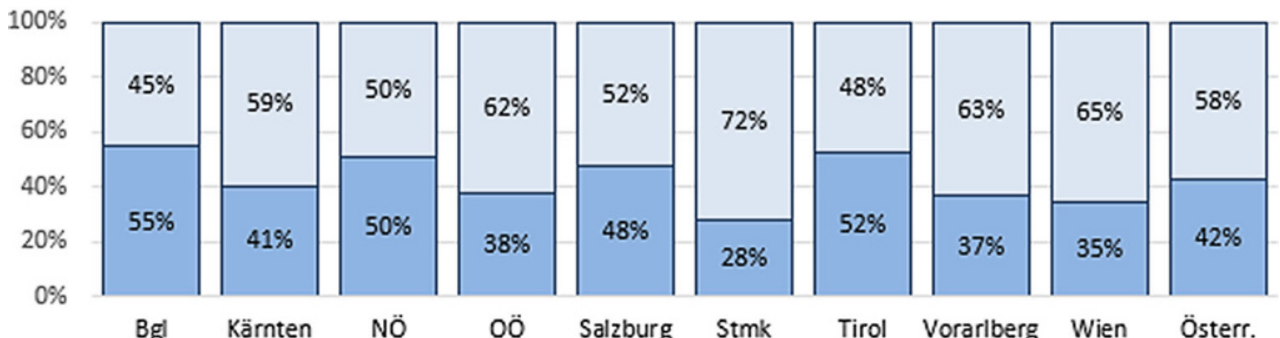

$\square$ Psycholog*innen

$\square$ Ärzt*innen regelung möglich, da in den nächsten Jahren nur mit einem sehr langsamen Zuwachs an Fachärzt*innen an den Abteilungen gerechnet werden kann.

Die meisten Bewerbungen für eine Assistentenstelle gibt es an den Universitätskliniken, an denen im Rahmen des Medizinstudiums Kinder- und Jugendpsychiatrie in ausreichender Stundenanzahl unterrichtet wird und auch die Möglichkeit des KlinischPraktischen Jahres gern in Anspruch genommen wird. Die weitere Integration des Faches in die universitäre Ausbildung ist damit eine wichtige Maßnahme, um den Studierenden das Fach bekannt zu machen und ihnen zu ermöglichen, sich dafür zu interessieren. Auch das Instrument des Status von Krankenanstalten als Lehrkrankenhäuser wäre zielführend, um zukünftigen Kollegen die aus den Bundesländern kommen, die regional nahen Abteilungen nahezubringen und für Ausbildung in KJPP zu gewinnen.

\section{Interdisziplinarität in der Kinder- und Jugendpsychiatrie}

Kinder- und jugendpsychiatrische Arbeit erfordert ein interdisziplinäres therapeutisches Team. Dazu bedarf es unterschiedlicher Berufsgruppen. Ein wichtiges Gegenüber sind die klinischen Psycholog*innen. Es sind insgesamt 130 Psycholog*innen (VZÄ) in den ambulanten und stationären kinder- und jugendpsychiatrischen Einrichtungen beschäftigt. In den meisten Abteilungen sind Psycholog*innen mit Fallführungsaufgaben betraut. Bei den stationären Einrichtungen sind meist mehr Ärzt*innen als bei den ambulanten Ein- 


\section{Erreichung der Vollversorgung in den unterschiedlichen Bereiche in $\%$}

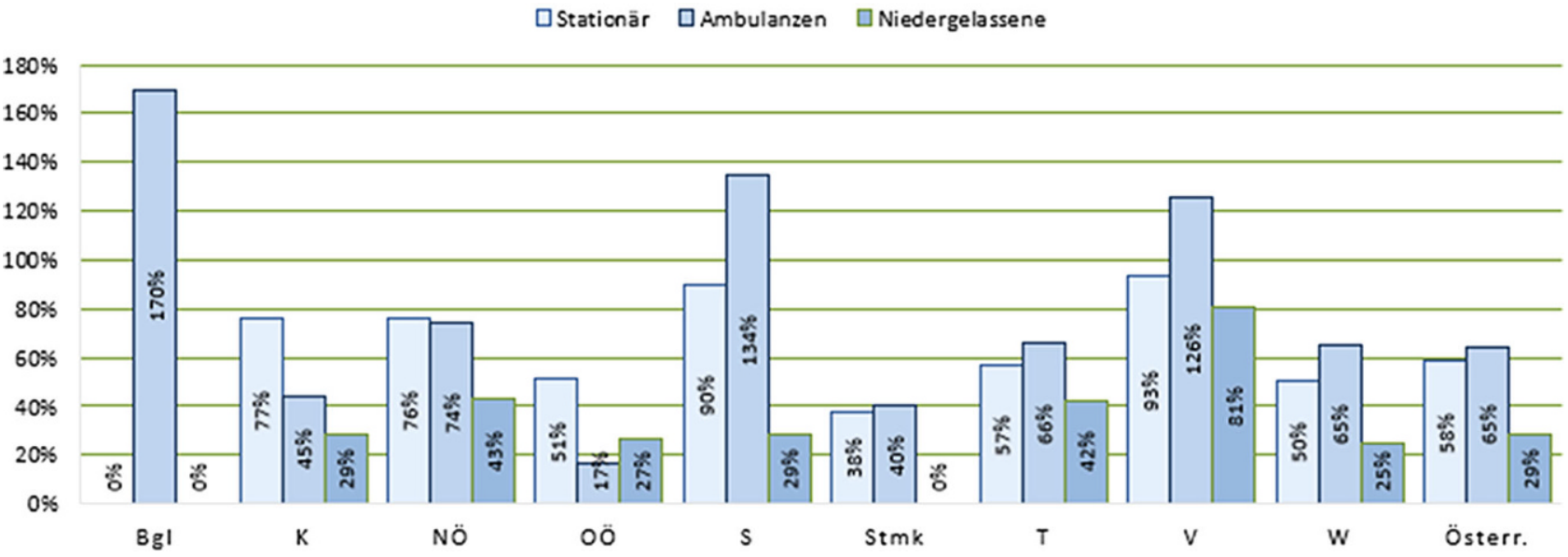

Abb. 8 Erreichung der Vollversorgung in den unterschiedlichen Bereichen in \% (2019)

richtungen tätig, da von den Ärzt*innen die Nachtdienste geleitet werden. Siehe auch Abb. 7.

\section{Kooperation, Transition und Nachhaltigkeit der Behandlung}

Die kinder- und jugendpsychiatrische Versorgung erfolgt in einer engen Kooperation und Vernetzung mit anderen medizinischen Fächern (Kinderheilkunde, Psychiatrie) und den nicht-medizinischen Säulen der Jugendhilfe, Sozialhilfe und den edukativen Bereichen (Kindergarten, Schule und Arbeitsmarktförderungen). Kooperationen und Übergänge in der Behandlung sind damit relevante Faktoren, sowohl für die Schaffung und den Erhalt des Zuganges zu einer kinder- und jugendpsychiatrischen Behandlung (Versorgungsprävalenz), als auch für die Erlangung einer nachhaltigen Wirksamkeit der Behandlungen.

In den Bundesländern wurden unterschiedliche Kooperationsprojekte mit Institutionen umgesetzt, die schwerpunktmäßig mit Kindern und Jugendlichen arbeiten, wie zum Beispiel die Kindernetzwerke. Es sind zudem spezifische Kooperationen zwischen Jugendhilfe und KJPP entstanden und verschiedene Ansätze der Kooperation zwischen Schule und KJPP.

\section{Vollversorgung als Ziel}

Ziel ist die bundesweite Vollversorgung im Sonderfach Kinder- und Jugendpsychiatrie und Psychotherapeutische Medizin. Abb. 8.

\section{Fazit}

Es besteht die Dynamik eines sich in Entwicklung befindlichen Sonderfaches der Medizin.

13 Jahre nach Gründung des Sonderfaches besteht weiterhin große Heterogenität der Versorgungslage zwischen den Bundesländern, sowohl auf der stationären als auch tagesklinischen und ambulanten/ niedergelassenen Ebene (Extrembeispiel „Steiermark, Wien“ vs. „Salzburg, Vorarlberg“). Um die Versorgung zu verbessern, muss die Sonderfachausbildung vorangetrieben werden, damit eine ausreichende Anzahl an Ärzt*innen ausgebildet wird. Die Mangelfachregelung in der derzeitigen Form ist nicht ausreichend, um die Ausbildung im Sonderfach zu konsolidieren und eine Vollversorgung in absehbarer Zeit zu erreichen. Dazu bedarf es dringend einer Bewegung von Seiten der Österreichischen Ärztekammer.

In die Leistungsorientierte Krankenhausfinanzierung LKF müssen auch mobile Angebote integriert werden, damit in Österreich moderne Behandlungsstrukturen geschaffen werden können, wie sie in Deutschland und der Schweiz zum Standard gehören.

Funding Open access funding provided by Medical University of Vienna.

Interessenkonflikt R. Fliedl, B. Ecker und A. Karwautz geben an, dass kein Interessenkonflikt besteht.

Open Access Dieser Artikel wird unter der Creative Commons Namensnennung 4.0 International Lizenz veröffentlicht, welche die Nutzung, Vervielfältigung, Bearbeitung, Verbreitung und Wiedergabe in jeglichem Medium und Format erlaubt, sofern Sie den/die ursprünglichen Autor(en) und die Quelle ordnungsgemäß nennen, einen Link zur Creative Commons Lizenz beifügen und angeben, ob Änderungen vorgenommen wurden.

Die in diesem Artikel enthaltenen Bilder und sonstiges Drittmaterial unterliegen ebenfalls der genannten Creative Commons Lizenz, sofern sich aus der Abbildungslegende nichts anderes ergibt. Sofern das betreffende Material nicht unter der genannten Creative Commons Lizenz steht und die betreffende Handlung nicht nach gesetzlichen Vorschriften erlaubt ist, ist für die oben aufgeführten Weiterverwendungen des Materials die Einwilligung des jeweiligen Rechteinhabers einzuholen.

Weitere Details zur Lizenz entnehmen Sie bitte der Lizenzinformation auf http://creativecommons.org/licenses/by/4. $0 /$ deed.de. 


\section{Literatur}

1. Fliedl R. Probleme der Primärversorgung in der Kinder- und Jugendpsychiatrie. Paediatr Paedolog Austria. 2013;48(Suppl 1):85-90.

2. Hartl C, Karwautz A. Zehn Jahre Kinder- und Jugendpsychiatrie in Österreich: ein neues ärztliches Sonderfach in den Strukturen des Gesundheitswesens. Neuropsychiatrie. 2017;31:103-11.https://doi.org/10.1007/s40211-0170235-0.

3. Fliedl R, Hart C, Karwautz A. Ist-Stand der Kinder- und Jugendpsychiatrischen Versorgung. 2018. https://oegkjp.at/ 2018-ist-stand-der-versorgung/.Zugegriffen: 17.11.2020.

4. Wagner G, Zeiler M, Waldherr K, Philipp J, Truttmann S, Dür W, et al. Mental health problems in Austrian adolescents: a nationwide, two-stage epidemiological study applying DSM-5 criteria. Eur Child Adolesc Psychiatry. 2017;26(12):1483-99. https://doi.org/10.1007/s00787017-0999-6.

5. Packard MA. Guideline to use of axis 5. 2011. https:// studylib.net/doc/8207667/guidelines-to-use- of-axis-v- global-assessment-functioning. Zugegriffen: 17.11.2020.

6. ÖGKJP. Versorgungsstrukturen für Kinder- und Jugendliche bei Psychischen Störungen und Krankheiten Leitlinien der ÖGKJP. 2004. https://oegkjp.at/wordpress/wpcontent/uploads/2017/12/2004_Versorgungsleitlinien. pdf.Zugegriffen: 17.11.2020.

7. Statistik Austria. Bevölkerungsanzahl Österreichs. 2020. https://www.statistik.at/wcm/idc/idcplg?IdcService=GET _PDF_FILE\&RevisionSelectionMethod=LatestReleased\&d DocName=023470.Zugegriffen: 17.11.2020.

8. Bednar W, Danzer D. Versorgungsstruktur Kinder- und Jugendneuropsychiatrie Wien. Österreichisches Bundesinstitut für Gesundheitswesen ÖBIG Planung Psychiatrie. 2004. https://oegkjp.at/wordpress/wp-content/uploads/
2017/12/EB-Planung-Psychiatrie-2004.pdf. Zugegriffen: 17.11.2020.

9. Bundesministerium für Soziales, Gesundheit, Pflege und Konsumentenschutz. LKF-Modell 2020 für den stationären Bereich. 2020f. https://www.sozialministerium. at/Themen/Gesundheit/Gesundheitssystem/Krankenan stalten/LKF-Modell-2020/Kataloge-2020.html. Zugegriffen: 17.11.2020.

10. ÖGKJP. Stellungnahme zur ambulanten Versorgung in der Kinder- und Jugendpsychiatrie (KJPP). 2018. https://oegkjp.at/wordpress/wp-content/uploads/2018/ 09/AmbulanteVersorgung2018.pdf.Zugegriffen:17.11.2020.

11. Walter R, Kampert K. Modellprogramm Psychiatrie. Forschungsschwerpunkt der Modellregion Marburg-B., Waldeck-F. und Schwalm-E. Marburg. 1986.

12. Statistik Austria. Bevölkerungsanzahl Österreichs. 2020. http://www.statistik.at/web_de/presse/122588.html\#: : text=Ihre\%20Zahl\%20erh\%C3\%B6hte\%20sich\%202019, 123.461\%20Menschen\%20am\%201.1.2020. Zugegriffen: 17.11.2020.

13. Statistik Austria. Bevölkerungsprognose 2019: Österreichs Bevölkerung wächst und altert weiterhin. 2019. https://www.statistik.at/web_de/statistiken/menschen_ und_gesellschaft/bevoelkerung/demographische_progno sen/bevoelkerungsprognosen/122070.html. Zugegriffen: 17.11.2020.

14. Statistik Austria. Bevölkerung zu Jahres- und Quartalsanfang. 2020. http://statistik.at/web_de/statistiken/ menschen_und_gesellschaft/bevoelkerung/bevoelkerungs stand_und_veraenderung/bevoelkerung_zu_jahres-_quar talsanfang/index.html.Zugegriffen: 17.11.2020.

Hinweis des Verlags Der Verlag bleibt in Hinblick auf geografische Zuordnungen und Gebietsbezeichnungen in veröffentlichten Karten und Institutsadressen neutral. 